\title{
High-resolution copy number array in the molecular cytogenetic diagnostics of pediatric malignant hematological disorders
}

\author{
CARINA WIKHAGER $^{1}$, INGER ÖGÄRD ${ }^{1}$, TOMMY MARTINSSON ${ }^{2}$, \\ JONAS ABRAHAMSSON $^{3}$, LARS PALMQVIST ${ }^{1}$ and HELENE SJÖGREN ${ }^{1}$ \\ Departments of ${ }^{1}$ Clinical Chemistry and Transfusion Medicine, and ${ }^{2}$ Clinical Genetics, Institute of Biomedicine, \\ University of Gothenburg, Sahlgrenska University Hospital, SE-41345 Gothenburg; ${ }^{3}$ Department \\ of Pediatrics, The Queen Silvia Children's Hospital, SE-41685 Gothenburg, Sweden
}

Received November 25, 2011; Accepted December 29, 2011

DOI: $10.3892 /$ or.2012.1689

\begin{abstract}
The presence of genetic alterations was investigated by SNP array in combination with conventional and spectral karyotyping and fluorescence in situ hybridization analysis of 75 consecutive pediatric bone marrow samples. The samples were collected at diagnosis from all children diagnosed with malignant hematological disease between 2006 and 2010 at a single diagnostic center in Gothenburg, Sweden. Conventional cytogenetic and molecular genetics techniques are up to this date essential for the clinical laboratories but there is a need for higher resolution techniques in order to identify new genetic markers that eventually can improve the management of these disorders. Here, we conclude that the addition of SNP array-based karyotyping combined with conventional cytogenetics increase the diagnostic accuracy of pediatric hematological malignancies. The two techniques enhance the cytogenetic image and should not be contrasted as they meet distinct important roles. Since balanced translocations cannot be detected with the SNP arrays of today and tumor-specific translocations are very important diagnostic and prognostic indicators we suggest that SNP-based array is a valuable adjuvant tool in the cytogenetic diagnostics of pediatric leukemias but cannot replace currently used techniques, i.e. G-banding, SKY and FISH analysis.
\end{abstract}

\section{Introduction}

The importance of genetic characterization with cytogenetic and molecular genetics methods is well-established in the diagnosis, prognosis prediction and determination of therapy

Correspondence to: Dr Helene Sjögren, Department of Clinical Chemistry and Transfusion Medicine, Institute of Biomedicine, University of Gothenburg, Sahlgrenska University Hospital, SE-41345 Gothenburg, Sweden

E-mail: helene.sjogren@vgregion.se

Key words: hematological malignancy, cytogenetics, aCGH in the management of malignant hematological disorders. However, there are limitations in the currently used techniques that need to be overcome. The methods used have limited detection sensitivity and resolution, and are also relatively expensive. Conventional cytogenetics are time-consuming; they are dependent on cell growth in vitro and good quality of metaphase spreads and have low resolution. Molecular genetics techniques, i.e. fluorescence in situ hybridization (FISH), and polymerase chain reaction (PCR) are sensitive and highly specific, but only for selected targets, and are also relatively labor-intensive, especially since the number of possible molecular markers that need to be investigated may be overwhelming. These factors make them both costly and inefficient. It is therefore important to introduce new techniques that can overcome these obstacles. There is also a need to identify new genetic markers that eventually can improve the management of these disorders. Microarray-based genomic profiling is one technique that may help to achieve this goal.

Different array based techniques have proven to be useful complementary tools in characterizing chromosomal aberrations in many tumor types $(1,2)$. Recently, high-density oligonucleotide based single nucleotide polymorphism (SNP) arrays have been used in the analysis of whole-genome copy number. SNP arrays, in combination with computational calculation algorithms, permit a more precise detection of common submicroscopic genomic lesions, amplifications, deletions, and allelic imbalances, such as copy number and neutral loss of heterozygosity (CNN-LOH) $(3,4)$. So far, comparative genomic hybridization arrays, aCGH, or SNP array analysis have mainly been used as a research tool but may now be ready to be introduced in clinical laboratory routines and could possibly replace some of the currently used laboratory procedures and improve the genetic characterization of malignant hematological neoplasms.

In order to validate the use of a genome-wide high resolution DNA copy number analysis in combination with conventional cytogenetic and molecular cytogenetic analysis, a SNP array (Affymetrix, GeneChip ${ }^{\circledR} 250 \mathrm{~K}$ Nsp) analysis was performed on 75 consecutive pediatric hematological malignancies diagnosed at the Sahlgrenska University Hospital, Gothenburg, Sweden between 2006 and 2010. This study shows that the SNP array could provide additional cytoge- 
netic information, more precise chromosome breakpoint information and could increase the detection rate of loss of the $C D K N 2 A$ gene $(9 \mathrm{p} 21)$.

\section{Materials and methods}

Patients. Bone marrow aspirates were consecutively collected from 75 children referred for diagnosis and treatment at The Queen Silvia Children's Hospital during the years 2006 and 2010 (Table I). All patients were diagnosed with malignant hematopoietic disorders, including 45 patients with precursor B lymphoblastic leukemia (B-ALL), 11 with T-cell acute lymphoblastic leukemia (T-ALL), 14 with acute myeloid leukemia (AML), two with myelodysplastic syndrome (MDS), one with chronic myeloid leukemia (CML), one with acute promyelocytic leukemia (APL) and one with Burkit's lymphoma (BL). All bone marrow samples were analyzed using conventional cytogenetic, molecular cytogenetic and molecular genetics techniques. In addition, all 75 cases were analyzed using SNP array. The study was performed in accordance to the Declaration of Helsinki and approval by the Ethics Committee, registration number KI02-560.

Cytogenetic, spectral karyotype (SKY) and fluorescence in situ hybridization (FISH) analyses. Conventional cytogenetics, SKY and FISH were performed according to standard procedures at the time of diagnosis. All bone marrow aspirates were cultured, harvested and slides were prepared and G-banded. Conventional chromosome G-banding and/ or SKY analysis was performed in all cases. The conditions for pretreatment, hybridization, post-hybridization washes, detection and analysis were essentially as recommended by the manufacturer (ASI-Applied Spectral Imaging Ltd., Migdal Ha'Emek, Israel) and as previously described (5). In most cases at least 20-25 metaphases were analyzed by two independent reviewers using a combination of G-banding and SKY. Karyotypes were described according to ISCN 2005 and 2009 (6,7). FISH analysis was performed on both metaphase and interphase cells using different probe panels depending on diagnosis. The FISH panel used for B-ALL and T-ALL include ETV6-RUNX1 [t(12;21)(p13;q22)], BCR-ABL1 [t(9;22) (q34;q11)], MLL (11q23), E2A (19p13), CDKN2A (9p21) and centromere specific probes for chromosomes 9 and 20 , for the identification of $\operatorname{dic}(9 ; 20)(\mathrm{p} 11 \sim 13 ; \mathrm{q} 11)$, for AML RUNX1-ETO [t(8;21)(q22;q22)], PML-RARA [t(15;17)(q22;q21)], CBFB [inv(16)(q22)], $M L L$ (11q23), for CML $B C R-A B L 1$, for APL $P M L-R A R A$, and for BL the $I G H-M Y C$ [t $(8 ; 14)(\mathrm{q} 24 ; \mathrm{q} 32)]$ probe. All probes are commercially available and purchased from Vysis (Downers Grove, IL) except for the probe for the $E 2 A$ gene which was purchased from Cytocell (Cambridge, UK). For each case at least 200 nuclei were scored by two independent reviewers.

DNA and RNA extraction and reverse transcription. DNA was extracted from $1 \times 10^{5}-5 \times 10^{6}$ leukocytes with a QIAamp DNA Blood Mini kit (Qiagen, Hilden, Germany) according to the instructions of the manufacturer. Total RNA was extracted using TRIzol reagent (Invitrogen, Carlsbad, CA). Firststrand cDNA was synthesized from $500 \mathrm{ng}$ total RNA using Superscript II reverse transcriptase (Invitrogen) and random
Table I. Diagnoses of the 75 cases from which bone marrow aspirates were obtained.

\begin{tabular}{lc}
\hline Diagnosis & No. of cases \\
\hline B-ALL & 45 \\
Hyperdiploid & 19 \\
t(12;21) & 10 \\
Other & 16 \\
T-ALL & 11 \\
AML & 14 \\
t(8;21) & 2 \\
inv(16) & 1 \\
der(11)(q23) & 4 \\
Other & 7 \\
MDS & 2 \\
CML & 1 \\
APL & 1 \\
BL & 1 \\
Total & 75 \\
\hline
\end{tabular}

hexamers in $20 \mu 1$ reactions according to the instructions of the manufacturer.

Reverse transcriptase-polymerase chain reaction (RT-PCR). Quantitative RT-PCR was performed with primer and probes according to consensus procedures (8), to verify the inversion of chromosome 16 ( $C B F B-M Y H 11)$, identify the $M L L$ fusion partner and to verify the FISH result RUNX1-ETO, RUNX1-ETV6, BCR-ABL1 and PML-RARA.

Single nucleotide polymorphism (SNP) arrays. The GeneChip ${ }^{\circledR}$ Human Mapping Affymetrix 250K (Affymetrix, Inc., Santa Clara, CA) array was used to perform a SNP array analysis. The $250 \mathrm{~K}$ array detects $\sim 262,000$ SNPs. All samples were compared in silico with healthy individuals. The array experiments were performed according to the protocol provided by the supplier (Affymetrix). Total genomic DNA (250 ng) was digested with the $N s p l$ restriction enzyme and ligated to adaptors. After ligation, the template was subjected to PCR amplification using a generic primer that recognizes the adaptor sequence. The amplified DNA was fragmented with DNase I, labelled with biotin and hybridized to the GeneChip Human Mapping 250K array. The hybridized probes were washed using the Affymetrix Fluidics Station 450 and marked with streptavidin-phytoerythrin. The arrays were scanned using a confocal laser scanner, GeneChip Scanner 3000 (Affymetrix).

Data analysis. The primary data analysis was performed using GDAS software (Affymetrix), while further studies were performed using CNAG software, version 3.0 (Genome Laboratory, Tokyo University, http://www.genome.umin. jp) $(9,10)$ and in NEXUS discovery (5.1) (Biodiscovery, El Segundo, CA). 
Table II. Value of the SNP array.

\begin{tabular}{lcrr}
\hline & & \multicolumn{2}{c}{ Was SNP of value? } \\
\cline { 3 - 4 } Diagnosis & No. of cases & Yes & No \\
\hline B-ALL & 45 & 27 & 18 \\
Hyperdipoid & 19 & 11 & 8 \\
t(12;21) & 10 & 5 & 5 \\
Other & 16 & 11 & 5 \\
T-ALL & 11 & 11 & 0 \\
AML & 14 & 2 & 12 \\
t(8;21) & 2 & 0 & 2 \\
inv(16) & 1 & 0 & 1 \\
der(11)(q23) & 4 & 1 & 3 \\
Other & 7 & 1 & 6 \\
MDS & 2 & 0 & 2 \\
CML & 1 & 1 & 0 \\
BL & 1 & 1 & 0 \\
APL & 1 & 0 & 1 \\
Total & 75 & 42 & 33 \\
\hline
\end{tabular}

\section{Results}

Clinical findings, cytogenetic and molecular genetic findings. The patient material consisted of 45 patients with B-ALL, 11 T-ALL, 14 AML, 2 MDS and 1 CML, APL and BL. The pediatric hematological material corresponds to the expected number of malignancies as well as the distribution of cytogenetic and genetic aberrations for this period at The Queen Silvia Children's Hospital. The karyotypic findings, based on G-banding and SKY, as well as the FISH and RT-PCR results were reported to the referring physician according to standard procedures.

The SNP array analysis identified gains, losses and CNN-LOH in all the acute leukemia types, except for in the APL. The SNP information showed different patterns in the different leukemia entities. Gains dominated the B-ALL patients and losses the T-ALL and AML patients. Nearly all of the CNN-LOH was detected in the T-ALL patients. In 42 $(56 \%)$ of the samples the SNP analysis was of value for the cytogenetic report, i.e. the array result added new information to the cytogenetic report and/or a cytogenetic finding was confirmed and/or a breakpoint was determined (Table II). All of the T-ALL patients took advantage of the array result while the AML patients did not gain almost any information by the technique. In the B-ALL material 27 of the 45 cases $(60 \%)$ showed an advantage of using the array. Altogether, the largest advantage of using SNP array was detected in the lymphocytic malignancies.

$B$-ALL. The 45 B-ALL patients included 19 patients showing karyotypes with modal numbers in the hyperdiploid to near-triploid region, 10 showing an ETV6-RUNX1/t $(12 ; 21)$ (p13;q22) translocation and 16 with other cytogenetic aberra- tions (Table I). As expected, the cytogenetically hyperdiploid cases showed whole chromosome gains using the SNP array. The concordance between the G-banding/SKY in these was high but in two cases the quality of the metaphases were poor and the array revised the karyotype. In another case a normal G-banding and FISH was reported but the array showed a hyperdiploid number of chromosomes. In about half of the hyperdiploid and the ETV6-RUNX1 positive leukemias there was added value of the SNP array while the 'other' cases showed added value in about $70 \%$ of the cases (Table II). The exact breakpoints could be determined in the karyotypes with unbalanced chromosome aberrations. In the cases with incomplete karyotypes (four cases) FISH analysis together with the SNP result gave additional cytogenetic information otherwise not identified. Twelve cases showed loss of CDKN2A (9p21) by FISH analysis as well as with SNP analysis. No additional losses of $C D K N 2 A$ were detected by SNP of the B-ALL patients. One of the hyperdiploid cases showed loss of one copy of the $C D K N 2 A$ gene as well as a $\operatorname{dic}(9 ; 20)$ translocation (Fig. 1). The translocation was seen by SKY and verified by a SNP array.

$T$-ALL. Cytogenetically, four T-ALL showed complex karyotypes, four a normal karyotype and two showed loss of chromosome material and one monosomy 21 . The T-ALL patients in this material showed loss of chromosome material. The four patients with apparently normal karyotypes showed 1-7 different losses of chromosome material each. Four areas of gained material were seen, including an extra copy of chromosomes $8,+8 \mathrm{q} 24,+9 \mathrm{q} 21-\mathrm{q} 34$ and $+13 \mathrm{q} 21$. In the cases with complex karyotypes the unbalanced translocations were verified and the breakpoints were determined. Loss of $C D K N 2 A$ using FISH was seen in four patients; these losses were verified with SNP that also detected four more. Two other patients showed CNN-LOH in the 9p24-p21 area. In total, 10 of 11 T-ALL had deletion of one or both copies of CDKN2A and/or CNN-LOH in the area, using the array technique.

$A M L$. The 14 AML patients were divided into two patients with a $R U N X 1-E T O / \mathrm{t}(8 ; 21)(\mathrm{q} 22 ; \mathrm{q} 22)$ translocation, one with an inversion of chromosome 16/CBFB-MYH11, four with aberrations in the $M L L$ gene (11q23) and seven patients showing other cytogenetic changes (Table I). A split signal for the MLL probe, using FISH analysis, was detected in all four cases and RT-PCR showed that these consisted of three $M L L-A F 9$ fusions and one $M L L-A F 10$ fusion. Most cytogenetic aberrations were identified by conventional cytogenetics and FISH and the SNP array did not contribute with additional cytogenetic information (Table II). In two cases (14\%), both with complex karyotypes, the array added cytogenetic information.

The two MDSs showed normal karyotypes and showed no aberrations with the SNP array (Table II). The CML showed a $B C R-A B L 1$ fusion using both FISH and RT-PCR analyses and the array showed extra material of chromosome 8 as well as loss of material from chromosome 12 . The BL showed a $\mathrm{t}(8 ; 22)$ (q24;q11) translocation and split signal for C-MYC (8q24) and the array showed several chromosome changes including both gains and losses. There were no signs of the $t(8 ; 22)(q 24 ; q 11)$ translocation indicating that the translocation was indeed 
A
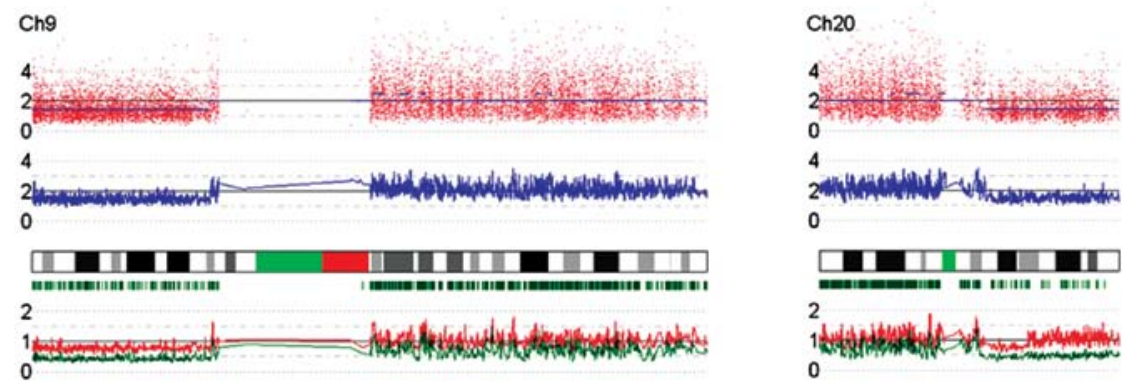

B

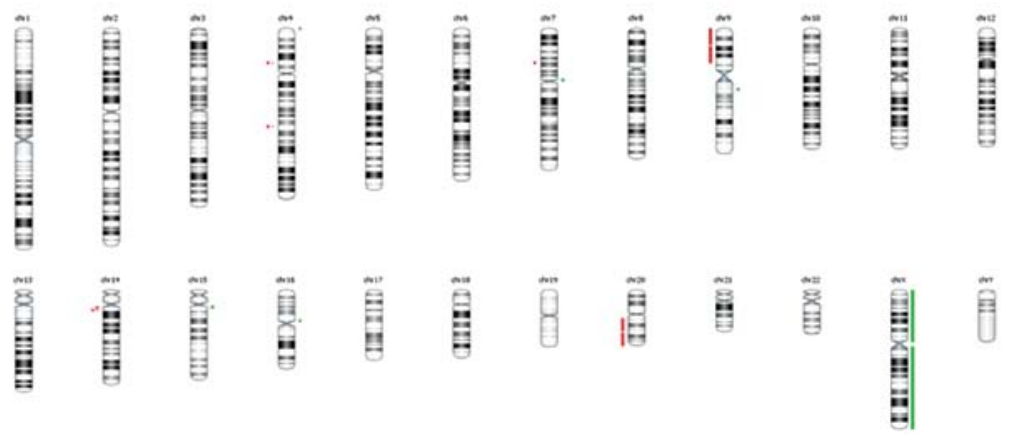

Figure 1. Dic $(9 ; 20)(\mathrm{p} 11 \sim 13 ; \mathrm{q} 11)$ seen by using (A) the CNAG and (B) the NEXUS software.

balanced. The APL had the characteristic $\mathrm{t}(15 ; 17)(22 ; \mathrm{q} 21)$ translocation and the PML-RARA fusion and the array did not show any additional changes.

\section{Discussion}

The presence of genetic alterations was investigated by SNP array in combination with conventional and spectral karyotyping and flourescence in situ hybridization analysis of 75 pediatric bone marrow samples. The samples were collected at diagnosis from all children diagnosed with malignant hematological disease between 2006 and 2010 at a single diagnostic center in Gothenburg, Sweden. Conventional cytogenetic and molecular genetics techniques are up to this date essential for the clinical laboratories but there is a need for higher resolution techniques in order to identify new genetic markers that eventually can improve the management of these disorders.

The SNP array analysis identified gains, losses and CNN-LOH in all the acute leukemia types, except for in the APL. The SNP information showed different patterns in the different leukemia entities. Gains dominated the B-ALL patients and losses the T-ALL and AML patients. In 42 (56\%) of the samples the SNP analysis was of value for the cytogenetic report, ie the array result added new information to the cytogenetic report and/or a cytogenetic finding was confirmed and/or a breakpoint was determined. The impact of the array data was particularly significant in the lymphocytic malignancies $(68 \%)$ while the myeloid malignancies did not gain as much information (17\%) by the technique (Table II).

The number of good quality metaphases to analyze is often low in ALL patients yet the genetic characterization is dependent on conventional G-banding and molecular cytogenetic techniques. It could be discussed if it is necessary to perform conventional G-banding for the T-ALL patients and the hyperdiploid B-ALL patients. FISH analysis, using the ALL FISH panel, together with the array data, covered all the cytogenetic aberrations in this T-ALL material as well as contributed with additional cytogenetic information. Flow cytometry analysis always precedes the G-banding analysis at this laboratory, thus giving the diploidity of the malignant cells indicating the hyperdiploid cases.

Moreover, the use of the FISH probe for analyzing suspected loss of the tumor suppressor gene CDKN2A (9p21) could also be debated. In this study 10/11 patients showed loss of $C D K N 2 A$ and only four of these were detected using FISH. The SNP array identified eight cases and two were detected using multiplex ligation-dependent probe amplification (MLPA). One problem with the FISH probe is that it covers around $222 \mathrm{~kb}$ and a very small deletion may occur within the probe and the signal pattern will appear normal. Five of the eleven T-ALL patients showed CNN-LOH in the 9p24-p13 area. It has been reported previously that loss of $C D K N 2 A$ is often seen in 9p CNN-LOH in pediatric T-ALL (11). Also, it has been shown that the pathophysiology of CNN-LOH may result in a similar gene expression pattern as in deletions (12). This proves to be a great disadvantage of the FISH technique for this particular probe and also it may suggest that there is a higher incidence of loss of $C D K N 2 A$ in pediatric T-ALL than what is reported. One reason why the probe for $C D K N 2 A$ is still used is for the detection for the $\operatorname{dic}(9 ; 20)$ translocation patients, as these show loss of one copy of $C D K N 2 A$ (13). One of the cases in this material showed a $\operatorname{dic}(9 ; 20)$ translocation (Fig. 1). The FISH pattern for these can be hard to interpret but both analysis programs for the array (CNAG and NEXUS) clearly showed the dic $(9 ; 20)$ translocation, which indicated the importance of this new technique. 
There is often a low number of copy number changes (CNCs) in the pediatric AML patients (14). In this study, all but one AML patients showed aberrations using one or the other technique. Most cytogenetic aberrations were identified by conventional cytogenetics as well as with SNP but six CNCs were added after the array. In the cases with a complex karyotype the unbalanced abnormalities were verified and the chromosomal breakpoints were determined. None of the balanced translocations were identified by the array. The results from data collected on the AML patients indicate that there is a value in performing both conventional routine techniques and SNP array but the gain of using SNP for the karyotype is not as obvious as for the ALL patients (Table II).

The number of MDS, CML, APL and BL patients were too low to comment but in order to find secondary chromosome aberrations and to further characterize these entities karyotypically SNP seems to be a good platform in combination with the conventional techniques. One of the CML patients showed extra material of chromosome $8(+8 \mathrm{p} 12-\mathrm{q} 24.3)$ as well as loss of material from chromosome 12 . Trisomy 8 emerges frequently at the time of acute transformation and is therefore important information.

In this study, the microarray-based genomic profiling technique (Affymetrix, GeneChip ${ }^{\circledR} 250 \mathrm{~K}$ Nsp) was used. The advantage of SNP compared to array CGH is that SNP can distinguish between the two alleles and can thus detect CNN-LOH, which has been shown to be of value not only in a clinical genetic settings, but also when studying acquired chromosome changes. A disadvantage with the SNP array platform is that the SNPs are not evenly distributed in the genome as opposed to the BAC (Bacterial Artificial Chromosome) arrays.

Today, there are Affymetrix platforms with higher resolution available than used in this article. The $250 \mathrm{~K}$ array improves the resolution of the cytogenetic information a lot $(15,16)$. The additional information gained by increased probe density was marginal, if any. In Mohamedali et al (15) patients with myelodysplastic syndromes were analyzed using $50 \mathrm{~K}$ SNP, 250K SNP and 500K SNP arrays. The concordance in the detection of uniparental disomies (UPD) were $100 \%$ and the $500 \mathrm{~K}$ array did not detect more regions of loss of heterozygosity than the $250 \mathrm{~K}$. The higher cost for the very high density arrays is maybe not in parity with the gained information.

There are a few issues to take in consideration when setting up array analysis in the routine diagnostic laboratory. It is important to remember that the cell population analyzed is a mixture of clonal and none clonal cells and that the cell populations are not purified. Some studies have shown that the sensitivity of purified cells is higher and others have shown that the gain is insignificant $(17,18)$. Also, when using DNA arrays it is important to bear in mind that if the clone constitutes less than $20 \%$ of cancer cells the aberrations can be undetectable. Sidelines and subclones are common in malignant neoplasms and there is no array information about these as the proportion of these cells is often too small. Another disadvantage to consider is that DNA arrays detect allelic dosage and therefore does not detect balanced translocations. Thus, conventional cytogenetics, FISH analysis and PCR techniques still must be performed according to standard protocols.

It is important clinically to distinguish between germline CNN-LOH, also called UPD and somatically acquired UPD
(19). UPD occurs when both copies of a chromosome pair, or part of a chromosome, originate from one parent (20). In this study, normal DNA samples were not available. Thus, it is impossible to know whether the UPD/CNN-LOH appears as a consequence of the malignancy or if it is a result of inheritance. Normal DNA reference material is needed in order to be able to distinguish between somatically acquired and inherited CNN variations or segments of homozygosity. In the future, it is desirable to compare the tumor DNA with a normal DNA sample (paired studies) at the time of diagnosis. It has been suggested that a skin biopsy would be the best choice of control DNA (21) and the most realistic may be DNA from saliva or buccal smears.

In the future, it is possible that second-generation DNA sequencing (or next generation sequencing) methods will enter the diagnostic arena and perhaps even replace the conventional cytogenetic methods as well as DNA array methods. Secondgeneration sequencing can possibly solve the analytic problem of tumor samples with a low tumor cell quantity, heterogeneity within the sample and variable ploidy. With this technique it will also be conceivable to analyze circulating cells which could be of future interest (22).

In conclusion, SNP array-based karyotyping combined with conventional cytogenetics increases the diagnostic accuracy of pediatric hematological malignancies. The two techniques enhance the cytogenetic image and should not be contrasted as they meet distinct important roles. Since balanced translocations cannot be detected with the SNP-arrays of today and tumor specific translocations are very important diagnostic and prognostic indicators we suggest that SNP-based array is a valuable adjuvant tool in the cytogenetic diagnostics of pediatric leukemias but cannot replace currently used techniques, i.e. G-banding and FISH.

\section{References}

1. Barrett MT, Scheffer A, Ben-Dor A, et al: Comparative genomic hybridization using oligonucleotide microarrays and total genomic DNA. Proc Natl Acad Sci USA 101: 17765-17770, 2004.

2. Pinkel D, Segraves R, Sudar D, et al: High resolution analysis of DNA copy number variation using comparative genomic hybridization to microarrays. Nat Genet 20: 207-211, 1998.

3. Gondek LP, Tiu R, O'Keefe CL, Sekeres MA, Theil KS and Maciejewski JP: Chromosomal lesions and uniparental disomy detected by SNP arrays in MDS, MDS/MPD, and MDS-derived AML. Blood 111: 1534-1542, 2008.

4. Heinrichs S, Kulkarni RV, Bueso-Ramos CE, et al: Accurate detection of uniparental disomy and microdeletions by SNP array analysis in myelodysplastic syndromes with normal cytogenetics. Leukemia 23: 1605-1613, 2009.

5. Sjogren H, Wedell B, Meis-Kindblom JM, Kindblom LG and Stenman G: Fusion of the $\mathrm{NH}_{2}$-terminal domain of the basic helix-loop-helix protein TCF12 to TEC in extraskeletal myxoid chondrosarcoma with translocation $\mathrm{t}(9 ; 15)(\mathrm{q} 22 ; \mathrm{q} 21)$. Cancer Res 60: 6832-6835, 2000.

6. Shaffer LG, Slovak ML and Cambell LJ (eds): ISCN 2009: An International System for Human Cytogenetic Nomenclature (2009). Karger, Basel, 2009.

7. Shaffer LG and Tommerup N (eds): ISCN 2005: An International System for Human Cytogenetic Nomenclature (2005): Recommendations of the International Standing Committee on Human Cytogenetic Nomenclature. Karger, Basel, 2005.

8. van Dongen JJ, Macintyre EA, Gabert JA, et al: Standardized RT-PCR analysis of fusion gene transcripts from chromosome aberrations in acute leukemia for detection of minimal residual disease. Report of the BIOMED-1 Concerted Action: investigation of minimal residual disease in acute leukemia. Leukemia 13: 1901-1928, 1999. 
9. Nannya Y, Sanada M, Nakazaki K, et al: A robust algorithm for copy number detection using high-density oligonucleotide single nucleotide polymorphism genotyping arrays. Cancer Res 65: 6071-6079, 2005.

10. Yamamoto G, Nannya Y, Kato M, et al: Highly sensitive method for genomewide detection of allelic composition in nonpaired, primary tumor specimens by use of Affymetrix single-nucleotide-polymorphism genotyping microarrays. Am J Hum Genet 81: 114-126, 2007.

11. Kawamata N, Ogawa S, Zimmermann M, et al: Molecular allelokaryotyping of pediatric acute lymphoblastic leukemias by high-resolution single nucleotide polymorphism oligonucleotide genomic microarray. Blood 111: 776-784, 2008.

12. Li X, Self SG, Galipeau PC, Paulson TG and Reid BJ: Direct inference of SNP heterozygosity rates and resolution of $\mathrm{LOH}$ detection. PLoS Comput Biol 3: e244, 2007.

13. Zachariadis V, Gauffin F, Kuchinskaya E, Heyman M, Schoumans J, Blennow E, Gustafsson B, Barbany G, Golovleva I, Ehrencrona H, et al; Nordic Society of Pediatric Hematology, Oncology (NOPHO); Swedish Cytogenetic Leukemia Study Group (SCLSG). The frequency and prognostic impact of $\operatorname{dic}(9 ; 20)(\mathrm{p} 13.2 ; \mathrm{q} 11.2)$ in childhood B-cell precursor acute lymphoblastic leukemia: results from the NOPHO ALL-2000 trial. Leukemia 4: 622-628, 2011.

14. Radtke I, Mullighan CG, Ishii M, et al: Genomic analysis reveals few genetic alterations in pediatric acute myeloid leukemia. Proc Natl Acad Sci USA 106: 12944-12949, 2009.

15. Mohamedali A, Gaken J, Twine NA, et al: Prevalence and prognostic significance of allelic imbalance by single-nucleotide polymorphism analysis in low-risk myelodysplastic syndromes. Blood 110: 3365-3373, 2007.
16. Maciejewski JP, Tiu RV and O'Keefe C: Application of arraybased whole genome scanning technologies as a cytogenetic tool in haematological malignancies. Br J Haematol 146: 479-488, 2009.

17. Gondek LP, Dunbar AJ, Szpurka H, McDevitt MA and Maciejewski JP: SNP array karyotyping allows for the detection of uniparental disomy and cryptic chromosomal abnormalities in MDS/MPD-U and MPD. PLoS One 2: e1225, 2007.

18. Starczynowski DT, Vercauteren S, Telenius A, et al: Highresolution whole genome tiling path array CGH analysis of CD $34^{+}$ cells from patients with low-risk myelodysplastic syndromes reveals cryptic copy number alterations and predicts overall and leukemia-free survival. Blood 112: 3412-3424, 2008.

19. Maciejewski JP and Mufti GJ: Whole genome scanning as a cytogenetic tool in hematologic malignancies. Blood 112: 965-974, 2008.

20. Tuna M, Knuutila S and Mills GB: Uniparental disomy in cancer. Trends Mol Med 15: 120-128, 2009.

21. Heinrichs S, Li C and Look AT: SNP array analysis in hematologic malignancies: avoiding false discoveries. Blood 115: 4157-4161, 2010.

22. Meyerson M, Gabriel S and Getz G: Advances in understanding cancer genomes through second-generation sequencing. Nat Rev Genet 11: 685-696, 2010. 